\title{
FUNDAMENTAL HLAWKA-LIKE INEQUALITIES FOR THREE AND FOUR VECTORS
}

\section{MARIUS MUNTEANU}

Abstract. We investigate Hlawka-like inequalities for three vectors and determine necessary and sufficient conditions such that

$$
a_{1} \sum_{i=1}^{3}\left\|x_{i}\right\|+a_{2} \sum_{1 \leqslant i<j \leqslant 3}\left\|x_{i}+x_{j}\right\|+a_{3}\left\|x_{1}+x_{2}+x_{3}\right\| \geqslant 0
$$

is satisfied for all $x_{1}, x_{2}, x_{3}$ in a Hlawka space. In addition, we show that any such inequality can be obtained as a linear combination with nonnegative coefficients of three fundamental inequalities, one of which is Hlawka's inequality.

In the case of four vectors in an inner product space, we prove that any (valid) inequality of the form

$$
a_{1} \sum_{i=1}^{4}\left\|x_{i}\right\|+a_{2} \sum_{1 \leqslant i<j \leqslant 4}\left\|x_{i}+x_{j}\right\|+a_{3} \sum_{1 \leqslant i<j<k \leqslant 4}\left\|x_{i}+x_{j}+x_{k}\right\|+a_{4}\left\|\sum_{i=1}^{4} x_{i}\right\| \geqslant 0
$$

can be written as a linear combination with nonnegative coefficients of six fundamental inequalities.

Mathematics subject classification (2010): 46C99, 47A30, 47A63.

Keywords and phrases: Hlawka's inequality.

\section{REFERENCES}

[1] D.D. Adamović, Généralisation d'une identité de Hlawka et de l'inegalite correspondante, Mat. Vesnik 116 (1964), 39-43.

[2] W. Berndt, S. SRA, Hlawka-Popoviciu inequalities on positive definite tensors, Linear Algebra Appl. 486 (2015), 317-327.

[3] D. Z. DJoković, Generalizations of Hlawka's Inequality, Glas. Mat.-Fiz. Astronom., Ser II, Društvo Mat. Fiz. Hratske, 18 (1963), 169-175.

[4] W. Fechner, Hlawka's functional inequality, Aequationes Math. 87 (2014), no. 1-2, 71-87.

[5] L. M. Kelly, D. M. Smiley, M.F. SMiley, Two dimensional spaces are quadrilateral spaces, Am. Math. Month. 72 (1965), 753-754.

[6] M. Munteanu, Extensions of Hlawka's inequality for four vectors, J. Math. Inequal. 13 (2019), no. 3, 891-901.

[7] D. S. Mitrinovici, Analytic Inequalities, Springer-Verlag, Berlin, 1970.

[8] D. M. Smiley, M. F. Smiley, The polygonal inequalities, Amer. Math. Monthly 71 (1964), 755 760.

[9] A. Simon, P. Volkmann, On Two Geometric Inequalities, Ann. Math. Sil. 9 (1995), 137-140.

[10] TAKAhashi, Y., WADA, S., TAKAhasi, S.-E., An extension of Hlawka's inequality, Math. Inequal. Appl. 3(1) (2000), 63-67.

[11] Witsenhausen, H.S., Metric inequalities and the zonoid problem, Proc. Am. Math. Soc. 40 (1973), $517-520$. 